\title{
Motor and molecular analysis to detect the early symptoms in a mouse amyotrophic lateral sclerosis model
}

\author{
Valeria Valsecchi ${ }^{*}$, Marina Boido, Antonio Piras, Giada Spigolon, Alessandro Vercelli \\ Neuroscience Institute Cavalieri Ottolenghi, Department of Neuroscience, University of Torino, Orbassano (TO), Italy; \\ ${ }^{*}$ Corresponding Author: valeria.valsecchi@unito.it
}

Received 21 July 2013; revised 21 August 2013; accepted 15 September 2013

Copyright (C) 2013 Valeria Valsecchi et al. This is an open access article distributed under the Creative Commons Attribution License, which permits unrestricted use, distribution, and reproduction in any medium, provided the original work is properly cited.

\begin{abstract}
The amyotrophic lateral sclerosis (ALS) is a progressive and fatal neurodegenerative disorder primarily involving motoneurons in the cerebral cortex, brainstem, and spinal cord. SOD1-G93A mice, which express multiple copies of the mutant form of the human Cu/Zn SOD, are one of the most widely used animal models for ALS pathology. However, the onset of the disease can vary between animals of 1 - 2 weeks while the progression is quite fast. In order to evaluate the efficacy of any treatment, it is very important to treat all animals at the early onset of the disease, instead of at a fixed age-point. To this aim, we performed behavioral analysis and measured hSOD1 mRNA expression to identify the appearance of the first motor deficits. Rotarod and PaGE tests revealed to be the most sensitive approaches to detect the beginning of the symptomatic phase of the disease, while neurological score and weight monitoring showed significant differences only at later stages in ALS pathology. Furthermore, we found a better correlation between hSOD1 mRNA expression with disease onset than with a transgene copy number. Therefore, the association of behavioral tests and molecular analysis represents a sensible and accurate tool to early detect the murine symptoms.
\end{abstract}

Keywords: SOD1-G93A Mice; Rotarod Test; PaGE Test; hSOD1 Expression

\section{INTRODUCTION}

Amyotrophic lateral sclerosis (ALS) is a fatal progres- sive neuromuscular disorder, with most patients dying within 3 to 5 years from diagnosis. Although most cases of ALS are sporadic in origin, $5 \%-10 \%$ are familial (FALS). Among the population with FALS, $10 \%$ possess a mutation in the gene that codes for the enzyme $\mathrm{Cu} / \mathrm{Zn}$ superoxide dismutase (SOD1) [1,2], an ubiquitously expressed protein that plays a key role in oxygen free radical scavenging, catalyzing the dismutation of superoxide $\left(\mathrm{O}_{2}^{-}\right)$to hydrogen peroxide $[3,4]$. Recently, other mutations involved in FALS cases have been discovered (TARDBP, FUS, C9ORG72,) [5]. Moreover, the finding of $C 9$ orf72 hexanucleotide repeat expansion in both sporadic and familial ALS suggests that many apparently sporadic cases are in fact underestimated familial cases $[6,7]$.

To investigate the pathogenic mechanisms leading to ALS, a number of animal models have been created based on genes involved in ALS pathogenesis [8]. However, the most widely used ALS experimental model remains the SOD1 mouse, even though it does not fully represent the human disease, since only few FALS cases are ascribed to its mutation, but better than others reproducing histopathological and clinical features of the disease. The SOD1 mouse was created by Gurney and colleagues in 1994 [9] by inserting a dominant "gain-of-function" mutation in human SOD1 over-expressed in the transgenic mice (the largest amount of mutant SOD is expressed in the brain). The mutation consists in the amino acid substitution at position 93 from glycine to alanine (G93A) that has little effect on enzyme activity.

The phenotype depends on the number of transgene copies [10,11]. Paralysis (in one or more limbs) is due to progressive loss of motoneurons. The mechanism by which the hSOD1-G93A mutation exerts a toxic effect is poorly understood. Multiple mechanisms have been proposed as causes of motoneuron degeneration including 
glutamate excitotoxicity [12], protein aggregation [13,14], increased oxidative stress, proteosome dysfunction $[15,16]$ and axonal transport defects $[17,18]$. The end-stage pattern of disease in these animals is the one most closely mimicking the changes that are generally observed in ASL patients, with filamentous inclusions in both neurons and axonal processes [19].

In order to evaluate the efficacy of any treatment, either pharmacological or cellular, it is very important to treat all animals at the effective early onset of the disease, at the same stage of progression. The outcome of treatment administered at a fixed age-point could result in misleading because of the variability of the onset ( 1 to 2 weeks). To this aim, we performed a set of three behavioral assessments (neurological condition, Rotarod and Paw Grip endurance tests) to evaluate motor performance that best correlates with the beginning of the symptomatic phase of the disease. Moreover, to explain different interindividual variance in the disease onset, we performed molecular analysis to measure hSOD1 mRNA expression levels in SOD1-G93A mice.

\section{MATERIALS AND METHODS}

\subsection{Animal Care and Use}

Male transgenic mice carrying the human mutated superoxide dismutase 1 gene (B6SJL-TgN[SOD1G93A]1 Gur, Jackson Laboratories; stock number 002726) were used; these mice have high transgene copy number, as reported in the datasheet. Founders were kindly gifted by M. Bentivoglio and R. Mariotti (University of Verona). The colony was derived by breeding male transgenic mice to naive $(\mathrm{B} 6 \mathrm{xSJL} / \mathrm{J}) \mathrm{F} 1$ females (Janvier SAS, Le GenestSaint-Isle, France).

All experimental procedures on live animals were performed according to the European Communities Council Directive of 24 November 1986 (86/609/EEC) and University of Torino's institutional guidelines on animal welfare (DL 116/92). All efforts were made to minimize the number of animals used and their suffering. After completion of the experimental procedure mice were overdosed with pentobarbital (i.p.) and euthanized by cervical dislocation.

\subsection{Genotyping Mice}

DNA from mouse tail was extracted incubating a small piece $(0.5 \mathrm{~cm})$ of tail in $100 \mu \mathrm{l}$ of lysis buffer $(10 \mathrm{mM}$ Tris $\mathrm{HCl}, 50 \mathrm{mM} \mathrm{KCl}, 0.01 \%$ gelatin, $0.45 \%$ IGEPAL, $0.4 \%$ Tween-20) and $25 \mu \mathrm{g}$ of proteinase $\mathrm{k}$ at $55^{\circ} \mathrm{C}$ over night under gentle shaking. On the extracted DNA we performed quantitative real-time PCR with a SYBR Green core reagent kit in a 7000 real time PCR system (Applied Biosystem) following the manufacturer's instructions in order to evaluate the presence and the copy number of the human transgene superoxide dismutase-1 (hSOD1). The primers used were:

5'-CATCAGCCCTAATCCATCTGA-3' and

5'-CGCGACTAACAATCAAAGTGA-3' for hSOD1 gene; 5'-CTAGGCCACAGAATTGAAAGATCT-3' and 5'-GTAGGTGGAAATTCTAGCATCATCC-3' for mouse interleukin 2 gene (mIL2), used as housekeeping gene [20]. Samples were amplified simultaneously in triplicate in 1 assay run. Changes in transgene copy number were determined as the difference in threshold cycle $(\Delta \mathrm{Ct})$ between the transgene and the reference gene (Ct hSOD1 - Ct mIL2).

\subsection{Evaluation of hSOD1 mRNA Expression}

Total RNA from 2-month-old mouse ears was extracted with Trizol following supplier's instructions (Invitrogen). The first-strand cDNA was synthesized with 10 $\mu \mathrm{g}$ of total RNA using the High Capacity cDNA Reverse Transcription Kit following supplier's instruction (Applied Biosystems). Quantitative real-time PCR was performed with SYBR green core reagent kit (Applied Biosystems). The primers used were

5'-AAGCATTAAAGGACTGACTGAAGG-3' and 5'-CACCGTGTTTTCTGGATAGAGG-3' to measure hSOD1 mRNA level;

5'-TGCACCACCAACTGCTTAGC-3' and 5'-GGCATGGACTGTGGTCATGAG-3' to measure glyceraldehyde 3-phosphate dehydrogenase (GADPH) mRNA, used as housekeeping gene for data normalization [21]. Samples were amplified simultaneously in triplicate in 1 assay run. Changes in mRNA levels were determined as the difference in threshold cycle $(\Delta \mathrm{Ct})$ between hSOD1 mRNA and the reference gene ( $\mathrm{Ct}$ hSOD1mRNA - $\mathrm{Ct}$ GAPDH).

\subsection{Behavioral Tests}

We performed the following behavioral tests: 1) neurological scoring of motor deficits by a trained observer; 2) performance on the Rotarod task; 3) Paw Grip Endurance $(\mathrm{PaGE})$; all of which are commonly used to evaluate SOD1-G93A animals [22,23]. Before starting the behavioral tests, the animals were weighed. Experiments were carried out following similar environmental conditions and at the same hour to reduce circadian influences. Beginning at P42 (postnatal day 42), the animals were assessed weekly, and around P80 (before the appearance of the first symptoms) twice per week. The first 2 weeks of tests were considered as training.

Neurological score: the mice were evaluated for signs of motor deficit with the following 4 point scoring system: 4 points if normal (no sign of motor dysfunction); 3 points if hind limb tremors were evident when suspended by the tail; 2 points if gait abnormalities were present; 1 
point for dragging of at least one hind limb; 0 points for inability to right itself within 30 seconds.

Rotarod test: we measured the time animals could remain on the rotating cylinder in a 7650 accelerating model of a Rotarod apparatus (Ugo Basile). Each animal was given three trials. The arbitrary cut-off time was 300 seconds.

Paw Grip Endurance (PaGE) test: the animal was placed on the wire-lid of conventional housing cage. The lid was gently shaken to prompt the mouse to hold onto the grid before it was swiftly turned upside down. Grip score was measured as the length of time that the mouse was able to hang on to the grid. The arbitrary cut-off time was 90 seconds.

\section{RESULTS}

\subsection{Behavioral Outcome}

For assessing the disease onset, 24 mice underwent a battery of behavioral tests: performance on the Rotarod, PaGE test, scoring of motor deficits by a neurological scale, and weighing. Two experienced investigators performed all behavioral tests.

The first two weeks of tests (starting around P60) were considered as training for the animals, and during this period mice were tested weekly until P80. Thereafter the tests were performed twice a week, to better monitor the animals and precisely detect the first symptoms.

Neurological test. This test was performed in an openfield in order to observe the mouse walking. The main problem of such test is that tremor phase (score 3) was not always evident: therefore it could happen to register for long score 4 (no motor dysfunctions), and then quickly switch to score 2 (gait abnormalities). However, as shown in the graph, this implies that animals displayed a normal posture until P111-P114 and suddenly worsened to 1.5 score in just one week (Figure 1(a)).

Rotarod test. This test measures motor performance and motor coordination of rodents. As showed in the graph, first training weeks were fundamental to animals for learning the task: in fact over the weeks the performance gradually improved and reached a plateau. The trend was quite stable until P100, when the motor performance started worsening. In about 25 days mice became totally unable to carry out the task (Figure 1(b)).

PaGE test. Animals were evaluated with this test to examine the hind limb resistance. Mice were able to finalize the test even without training, probably because this motor activity results more common than Rotarod for rodents. However, similarly to the Rotarod, PaGE test highlighted a sudden worsening in the motor performance from P100, until they were completely unable to perform the task around P128 (Figure 1(c)).

Body weight. We observed a body weight difference between P90 (when animals are adult and ALS onset is not yet evident, as shown by the other tests) and P128 (late-phase of the disease). The weight showed only a modest decrease (about 6\%) in the last month (Figure 1(d)).

\subsection{Correlation between SOD1 mRNA and Disease Onset}

Four animals, out of the 24 analyzed at the behavioural tests, were excluded from the average because they showed the first symptoms of the disease early in advanced compared to the other 20 mice (mice number 1-4, Figure 2). These four animals showed the first symptoms of the disease between P70 and P90, approximately from 20 to 40 days before the average value observed with the other 20 mice (P110).

It has been previously reported that changes in transgene copy number in SOD1-G93A mice can sometimes occur after mating, due to intra locus recombination events, usually resulting in delayed disease onset and an increase in the lifespan of these animals [24].

Therefore, using real time quantitative PCR, we measured the relative transgene copy number in the 4 "atypical" mice, and in 6 mice that showed the first symptoms of the disease approximatively at P110, to investigate the correlation between age of disease onset and transgene copy number (Figure 2(a)). From the ear of the same animals we measured mRNA expression levels of hSOD1 gene at 2 months of age (Figure 2(b)). The ear was chosen because in preliminary experiments (data not shown) we extracted RNA from different tissue samples (ear, spinal cord, cortex, cerebellum, tail, fingers) and we observed a direct correlation in hSOD1 mRNA expression between spinal cord and ear.

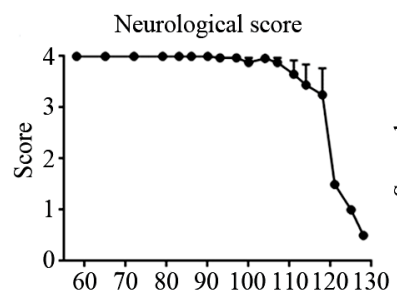

(a)

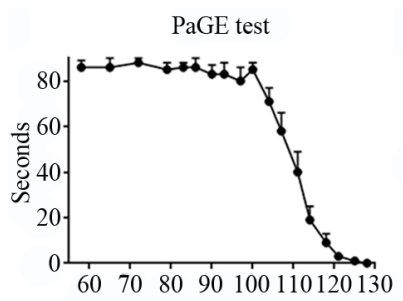

(c)

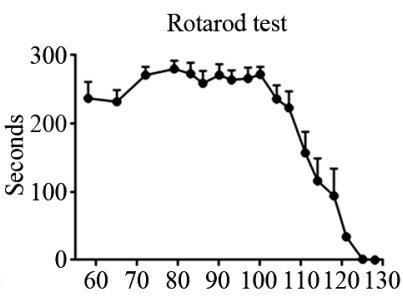

(b)

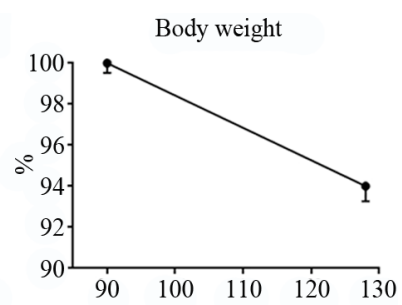

(d)
Figure 1. Panel A, neurological score of SOD1-G93A mice. Panel B-C, Rotarod and PaGE behavioral tests, respectively. Panel D, weight loss in SOD1-G93A mice. 


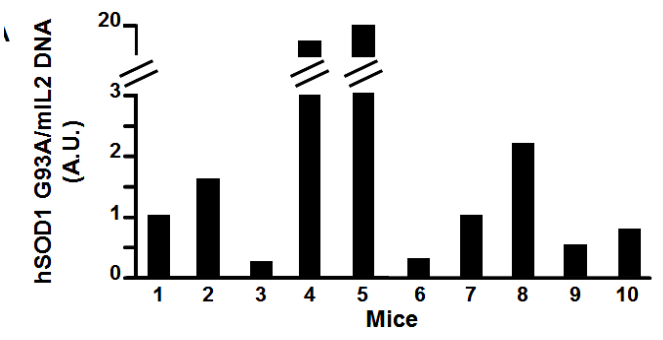

(a)

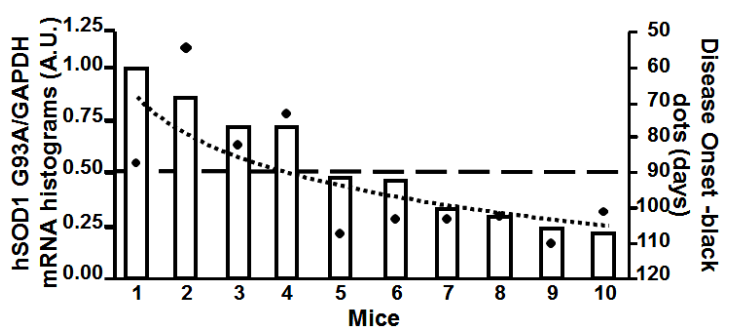

(b)

Figure 2. Panel A, hSOD1 gene copy number evaluation in SOD1-G93A mice. Panel B, hSOD1 mRNA expression levels in SOD1-G93A mice (histograms) correlated to disease onset expressed in days (black dots).

We arbitrarily considered that, when the mouse showed behavioral deficits in at least two tests (i.e. values $<90$ seconds the PaGE test, $<300$ seconds in the rotarod test, and score $<4$ in the neurological test) for two consecutive measurements, such date corresponded to the disease onset.

Interestingly, our results showed a better correlation between disease onset and mRNA levels than with transgene copy number (Figure 2(b)). In particular, the "atypical" mice number 1, 2, 3 and 4, that showed the first symptoms of the disease earlier than P110, had a hSOD1 mRNA relative level greater than 0.5 (arbitrary unit scale; $\Delta \mathrm{Ct}>-3.5$ ). The remaining mice, with a relative value of hSOD1 mRNA lower than 0.5 , approximately around $0.25(\Delta \mathrm{Ct}<-2.5)$, showed the onset of the disease ranging from P100 to P110 as we would expect from this transgenic model. On the other hand, mice number 5, 6, 7 and 8 with quite different transgene copy number (arbitrary unit scale) showed approximately the same age of onset of the disease, around P110. This molecular analysis let us to characterize and to exclude from further studies the animals (mice number 1, 2, 3 and 4) with a too early onset of the disease.

\section{DISCUSSION}

One of the most widely used animal models of ALS consists in SOD1-G93A mice, which express multiple copies of the mutant form of the human $\mathrm{Cu} / \mathrm{Zn}$ SOD [24]. However a substantial problem in SOD1-G93A mice is represented by changes in transgene copy number occurring after mating, due to intra locus recombination events: this can result in delayed disease onset and an increase in their lifespan [25]. However, some authors refer to rarely detect the copy number reduction [26].

The expression level of mutated SOD1 can vary independently from the number of copies of the transgene, thus modifying the onset and progression of the disease. In fact, the appearance of the first symptoms varies among the animals, in a time window of $1 / 2$ weeks. Afterwards, the progression of the disease is quite fast, and the animals die approximately in 30 days. Due to the variability of disease onset, preclinical studies should rely on assessment for the precise detection of the first symptoms, instead of starting treatment in each animal at the same pre-defined age-point. Therefore, we used two different approaches, behavioral tests and molecular analysis to evaluate motor functions of male mice, in order to establish the beginning of the disease. We used only male animals, since ALS is characterized by a faster progression in males than in females probably for the partial protective role of estrogens in the latter ones [27]: in previous experiments, we have observed significant differences in terms of survival, motor performance and histological parameters between sexes, according to the literature [23].

In our study, the battery of behavioral tests performed on 24 mice revealed that the Rotarod and the PaGE tests were reliable markers of the onset of the disease.

The assessment of Rotarod performance has widely been used in various models of neurodegenerative diseases to monitor motor coordination, strength and balance: in fact, it is considered one of the most sensitive method for detecting symptoms in hemiplegic and ALSaffected mice [23,28,29].

In our experience, after the first weeks of training, mice can easily perform 300 seconds on the rotating cylinder, until the hind limbs show the first symptoms of weakness [30,31].

Similarly, mice perform very well at the Grip test at the first observation age point ( 6 weeks of age). They can resist upside down for long, but a cut-off of 90 seconds was set. Additionally we considerably concluded the test even though the mice kept on hanging with the only forelimbs for more than 5 seconds. In this way, we can distinguish between the strength of the forelimbs (involved later in disease) and the hind limbs. Furthermore, the Grip test is less expensive and less time consuming than the Rotarod apparatus, and does not require a training, even though we advise to accustom mice with the test.

Additionally, in contrast with the large number of animals considered, SEM for the Rotarod test was considerably higher than for the PaGE test; therefore, the latter displays a minor variability and a better reliability.

On the other hand, the neurological score evaluation 
and the body weight loss gave poor results. In particular, only when the disease has been already in an advanced phase (approximately P115 days), we observed a significant decrease in the neurological score and in the weight, according to Knippenger and Coll. (2010) [29] who detected first abnormalities around P109. Moreover, the visual evaluation (neurological score) by an experienced investigator is not sufficient for identifying the first symptoms, since particularly during the first stages, mice appear quite unaltered although the motoneuron degeneration is already ongoing [29].

Additionally, we can not easily explain why sometimes our animals switched straight from a 4 point score (no motor dysfunction) to gait abnormalities (score 2). For all these reasons, we consider that this test is not suitable for an accurate evaluation of the onset of the disease. Nevertheless, for further information, we also report the Weydt's paper [22] which on the contrary, considers this kind of observation as the most sensitive method for detecting abnormalities.

Similarly, monitoring of the weight loss of the animals is poorly helpful in our opinion as previously reported by Knippenberg [29]. Probably, the decrease becomes lately evident, due to the difficulty in feeding as ALS related-muscular atrophy occurs. Pharmacological treatment of animals at this stage of the disease can unlikely give encouraging results. Therefore, weight and neurological score can only be considered additional parameters to monitor disease progression.

Furthermore, we measured transgene copy number and mRNA expression levels of hSOD1 in 10 out of 24 mice analyzed at the behavioral tests. We considered 4 "atypical" mice that showed the first symptoms of the disease early in advance compared to the other mice, and 6 of the "typical" mice. Interestingly, we observed a better correlation between the onset of the disease and hSOD1 mRNA expression levels in two month-old mice ears, rather than to the transgene copy number.

The inconsistence between hSOD1 mRNA expression and gene copies can be explained by the mislocation of the transgene in a poorly transcribed region. Therefore, the transgene copy number can be a misleading parameter. In fact, even high transgene copy number can produce low mRNA hSOD1 levels in the mouse, corresponding to a late-onset disease (mouse number 5 in Figure 2). By the contrary, mice with low transgene copy number, probably in a highly transcribed region, can develop the disease quite early (mouse number 3 in figure 2). In particular, mice with a relative mRNA level below 0.5 (arbitrary unit) will be considered suitable for further pharmacological experiments whereas mice with higher mRNA levels will be excluded for a too premature disease onset.

For a pharmacological study, it is very important to have a homogenous group of animals (all at the initial phase of the disease). However, sometimes the symptoms onset cannot be arbitrarily fixed; therefore, we employ the behavioral tests for precisely detecting the first symptoms, and we specifically consider this moment (i.e. when the mouse shows at least two behavioral deficits for two consecutive measurements) as the exact disease onset. Simply referring to the animal age could in fact generate unreliable data.

Such approach allows to clearly identify the effects of the treatment on animal behavior and their lifespan: in fact a decrease in the progression of the disease or an increase in the lifespan of the mice of 1 or 2 weeks would represent a very encouraging result, due to short lifespan of this animal.

In conclusion, we show that i) a battery of behavioral tests allows precisely detecting the first symptoms, and the onset of the disease; and ii) there is a direct correlation between hSOD1 mRNA expression and the age of onset of the disease.

The association of behavioral tests and molecular analysis represents a sensible and accurate tool to early detect the murine ALS symptoms and to perform a pharmacological treatment in the best therapeutic window.

\section{ACKNOWLEDGEMENTS}

This work was supported by Italian Ministry of University and Research (MIUR) grant to A.V.

\section{REFERENCES}

[1] Siddique, T., Figlewicz, D.A., Pericak-Vance, M.A., Haines, J.L., Rouleau, G., Jeffers, A.J., Sapp, P., Hung, W.Y., Bebout, J., McKenna-Yasek, D. et al. (1991) Linkage of a gene causing familial amyotrophic lateral sclerosis to chromosome 21 and evidence of genetic-locus heterogeneity. The New England Journal of Medicine, 324, 1381-1384. http://dx.doi.org/10.1056/NEJM199105163242001

[2] Rosen, D.R., Siddique, T., Patterson, D., Figlewicz, D.A., Sapp, P., Hentati, A., Donaldson, D., Goto J., O'Regan, J.P., Deng, H.X., et al. (1993) Mutation in $\mathrm{Cu} / \mathrm{Zn}$ superoxide dismutase gene are associated with familial amyothrophic lateral sclerosis. Nature, 362, 59-62.

[3] Tainer, J.A., Getzoff, E.D., Beem, K.M., Richardson, J.S. and Richardson, D.C. (1982) Determination and analysis of the 2 A-structure of copper, zinc superoxide dismutase. Journal of Molecular Biology, 160, 181-217. http://dx.doi.org/10.1016/0022-2836(82)90174-7

[4] Scozzafava, A. and Viezzoli, M.S. (1993) The role of the active site amino acid residues on the catalytic activity of $\mathrm{Cu} 2 \mathrm{Zn2SOD}$. Molecular and Chemical Neuropathology, 19, 193-204. http://dx.doi.org/10.1007/BF03160179

[5] Ince, P.G., Highley, J.R., Kirby, J., Wharton, S.B., Taka- 
hashi, H., Strong, M.J. and Shaw, P.J. (2011) Molecular pathology and genetic advances in amyotrophic lateral sclerosis: An emerging molecular pathway and the significance of glial pathology. Acta Neuropathologica, 122, 657-671. http://dx.doi.org/10.1007/s00401-011-0913-0

[6] Al-Chalabi, A., Jones, A., Troakes, C., King, A., Al-Sarraj, S. and van den Berg, L.H. (2012) The genetics and neuropathology of amyotrophic lateral sclerosis. Acta Neuropathologica, 124, 339-352.

http://dx.doi.org/10.1007/s00401-012-1022-4

[7] Majounie, E., Renton, A.E., Mok, K., Dopper, E.G., Waite, A., Rollinson, S., Chiò, A., Restagno, G., Nicolaou, N., Simon-Sanchez, J. et al. (2012) Frequency of the C9orf72 hexanucleotide repeat expansion in patients with amyotrophic lateral sclerosis and frontotemporal dementia: A cross-sectional study. The Lancet Neurology, 11, 323-330. http://dx.doi.org/10.1016/S1474-4422(12)70043-1

[8] Boido, M., Buschini, E., Piras, A., Spigolon, G., Valsecchi, V., Mazzini, L. and Vercelli, A. (2012) Advantages and pitfalls in experimental models of ALS. In: Maurer M.H., Ed., Amyotrophic Lateral Sclerosis, Chapter 5, InTech, 125-140. http://dx.doi.org/10.5772/31380

[9] Gurney, M.E., Pu, H., Chiu, A.Y., Dal Canto, M.C., Polchow, C.Y., Alexander, D.D., Caliendo, J., Hentati, A., Kwon, Y.W., Deng, H.X., Chen, W., Zhai, P., Sufit, R.L. and Siddique, T. (1994) Motor neuron degeneration in mice that express a human $\mathrm{Cu}, \mathrm{Zn}$ superoxide dismutase. Science, 264, 1772-1775.

[10] Chiu, A.Y., Zhai, P., Dal Canto, M.C., Peters, T.M., Kwon, Y.W., Prattis, S.M. and Gurney, M.E., (1995) Age-dependent penetrance of disease in a transgenic mouse model of familial amyotrophic lateral sclerosis. Molecular and Cellular Neuroscience, 6, 349-362.

http://dx.doi.org/10.1006/mone.1995.1027

[11] Dal Canto, M.C. and Gurney, M.E. (1997) A low expressor line of transgenic mice carrying a mutant human $\mathrm{Cu}$, Zn superoxide dismutase (SOD1) gene develops pathological changes that most closely resemble those in human amyotrophic lateral sclerosis. Acta Neuropathologica, 93, 537-550.

http://dx.doi.org/10.1007/s004010050650

[12] Pasinelli, P., Brown, R.H. (2006) Molecular biology of amyotrophic lateral sclerosis: Insights from genetics. $\mathrm{Na}$ ture Reviews Neuroscience, 7, 710-723. http://dx.doi.org/10.1038/nrn1971

[13] Hirano, A., Donnenfeld, H., Sasaki, S. and Nakano, I. (1984) Fine structural observations of neurofilamentous changes in amyotrophic lateral sclerosis. Journal of Neuropathology \& Experimental Neurology, 43, 461-470. http://dx.doi.org/10.1097/00005072-198409000-00001

[14] Shaw, B.F. and Valentine, J.S. (2007) How do ALS-associated mutations in superoxide dismutase 1 promote aggregation of the protein? Trends in Biochemical Sciences, 32, 78-85. http://dx.doi.org/10.1016/j.tibs.2006.12.005

[15] Kato, S., Saeki, Y., Aoki, M., Nagai, M., Ishigaki, A., Itoyama, Y., Kato, M., Asayama, K., Awaya, A., Hirano, A. and Ohama, E. (2004) Histological evidence of redox system breakdown caused by superoxide dismutase 1 (SOD1) aggregation is common to SOD1-mutated motor neurons in humans and animal models. Acta Neuropathologica, 107, 149-158.

http://dx.doi.org/10.1007/s00401-003-0791-1

[16] Schwartz, A.L. and Ciechanover, A. (1999) The ubiquitinproteasome pathway and pathogenesis of human diseases. Annual Review of Medicine, 50, 57-74.

http://dx.doi.org/10.1146/annurev.med.50.1.57

[17] Ström, A.L., Gal, J., Shi, P., Kasarskis, E.J., Hayward, L.J. and Zhu, H. (2008) Retrograde axonal transport and motor neuron disease. Journal of Neurochemistry, 106, 495505. http://dx.doi.org/10.1111/j.1471-4159.2008.05393.x

[18] Zhang, F., Ström, A.L., Fukada, K., Lee, S., Hayward, L.J. and Zhu, H. (2007) Interaction between familial amyotrophic lateral sclerosis (ALS)-linked SOD1 mutants and the dynein complex. The Journal of Biological Chemistry, 282, 16691-16699. http://dx.doi.org/10.1074/jbc.M609743200

[19] Hirano, A. (1991) Cytopathology of amyotrophic lateral sclerosis. Advances in Neurology, 56, 91-101.

[20] Alexander, G.M., Erwin, K.L., Byers, N., Deitch, J.S., Augelli, B.J., Blankenhorn, E.P. and Heiman-Patterson, T.D. (2004) Effect of transgene copy number on survival in the G93A SOD1 transgenic mouse model of ALS. Molecular Brain Research, 130, 7-15. http://dx.doi.org/10.1016/j.molbrainres.2004.07.002

[21] Zinman, L., Liu, H.N., Sato, C., Wakutani, Y., Marvelle, A.F., Moreno, D., Morrison, K.E., Mohlke, K.L., Bilbao, J., Robertson, J. and Rogaeva, E. (2009) A mechanism for low penetrance in an ALS family with a novel SOD1 deletion. Neurology, 72, 1153-1159. http://dx.doi.org/10.1212/01.wnl.0000345363.65799.35

[22] Weydt, P., Hong, S.Y., Kliot, M. and Möller, T. (2003) Assessing disease onset and progression in the SOD1 mouse model of ALS. Neuroreport, 23, 1051-1054.

[23] Vercelli, A., Mereuta, O.M., Garbossa, D., Muraca, G., Mareschi, K., Rustichelli, D., Ferrero, I., Mazzini, L., Madon, E. and Fagioli, F. (2008) Human mesenchymal stem cell transplantation extends survival, improves motor performance and decreases neuroinflammation in mouse model of amyotrophic lateral sclerosis. Neurobiology of Disease, 31, 395-405.

http://dx.doi.org/10.1016/j.nbd.2008.05.016

[24] Dal Canto, M.C., Gurney, M.E. (1994) Development of central nervous system pathology in a murine transgenic model of human amyotrophic lateral sclerosis. American Journal of Pathology, 145, 1271-1279.

[25] Gurney, M.E. (1997) The use of transgenic mouse models of amyotrophic lateral sclerosis in preclinical drug studies. Journal of the Neurological Sciences, 152, S67-S73. http://dx.doi.org/10.1016/S0022-510X(97)00247-5

[26] Mead, R.J., Bennett, E.J., Kennerley, A.J., Sharp, P., Sunyach, C., Kasher, P., Berwick, J., Pettmann, B., Bat- taglia, G., Azzouz, M., Grierson, A. and Shaw, P.J. (2011) Optimised and rapid pre-clinical screening in the SOD1 (G93A) transgenic mouse model of amyotrophic lateral sclerosis (ALS). PLoS One, 6, Article ID: E23244. http://dx.doi.org/10.1371/journal.pone.0023244

[27] Choi, C.I., Lee, Y.D., Gwag, B.J., Cho, S.I., Kim, S.S. 
and Suh-Kim, H. (2008) Effects of estrogen on lifespan and motor functions in female hSOD1 G93A transgenic mice. Journal of the Neurological Sciences, 268, 40-47. http://dx.doi.org/10.1016/j.jns.2007.10.024

[28] Ikeda, R., Kurokawa, M.S., Chiba, S., Yoshikawa, H., Ide, M., Tadokoro, M., Nito, S., Nakatsuji, N., Kondoh, Y., Nagata, K., Hashimoto, T. and Suzuki, N. (2005) Transplantation of neural cells derived from retinoic acidtreated cynomolgus monkey embryonic stem cells successfully improved motor function of hemiplegic mice with experimental brain injury. Neurobiology of Disease, 20, 38-48. http://dx.doi.org/10.1016/j.nbd.2005.01.031

[29] Knippenberg, S., Thau, N., Dengler, R. and Petri, S. (2010) Significance of behavioural tests in a transgenic mouse model of amyotrophic lateral sclerosis (ALS).
Behavioural Brain Research, 213, 82-87. http://dx.doi.org/10.1016/j.bbr.2010.04.042

[30] Durand, J., Amendola, J. and Bories, C. (2006) Lamotte d'Incamps B. Early abnormalities in transgenic mouse models of amyotrophic lateral sclerosis. Journal of Physiology-Paris, 99, 211-220.

http://dx.doi.org/10.1016/j.jphysparis.2005.12.014

[31] Mancuso, R., Oliván, S., Osta, R. and Navarro, X. (2011) Evolution of gait abnormalities in SOD1 (G93A) transgenic mice. Brain Research, 1406, 65-73. http://dx.doi.org/10.1016/j.brainres.2011.06.033 